\title{
THE LOGIC OF KNOWLEDGE
}

\author{
Nicholas Nisbet $^{1}$
}

\section{Background and Identification of Problem / Knowledge GaP}

Construction and the built environment sector has often be represented as being functionally unsustainable and inherently inefficient. External judgements are made on its poor financial stability, poor environmental performance and social failure. Internal assessments focus on poor satisfaction of client requirements, regulatory compliance and a failure to learn from recommendations and advisory material. This paper is intended to address the problem of failure to accumulate and apply knowledge systematically, leaving the sector dependant on tacit knowledge, experience and habits. There are plenty of research publication outlining compliance checking solutions based on labour-intensive programming of relatively specific problems, their accumulation into desktop tools (Solibri 1998 onwards) and one example of an integrated solution. The Singapore Governments ePlanCheck (1998-2004) system demonstrated that by bringing together regulatory domain experts, BIM schema experts and procedural coding skills, a solution was developed that accurately checked design proposals against the then current zoning, spatial and architectural building compliance regulations. The apparent success of this implementation led to optimism that other regulations regime could be addressed in the USA, Norway and UK. Successful proof-of-concepts were made for Scottish apartmentnoise performance regulations, and of delivering prioritised advice on mitigating the risk of falls for the UK Health and Safety Executive. The reality was that the solution failed to address two key success criteria: efficiency and acceptability. Requirements are specific to an individual project, and regulations are revised on a 2-5 yearly cycle. It would never be feasible to apply the three-party resources used in the Singapore project continuously to maintain, letalone extend, the scope. In the USA building code compliance is specified and administered at the level of 3000 independent counties. The acceptability of the results was also challenged in that there was no demonstrable connection between the authoritative text of the regulations and the results. Whilst other sectors, such a finance and medicine, are able to apply considerable resources to individual sub-problems, construction needs a generic approach so as to exploit the limited investment in process improvement and R\&D effectively. Operable knowledge is the knowledge that can be used systematically to drive design and engineering development and to the verify compliance systematically. The knowledge gap is how to render knowledge operable.

\section{Research Aim And Methodology}

AEC3 recognised that the problem could not be solved incrementally for example by gradual improvements in the style of source normative material, the adoption of different coding languages nor the simplification of the information models being presented. Regulatory experts were attempting to place key phrases into a regular spreadsheet structure. The structure of these spreadsheets seemed constantly expanding. Section, paragraph and sentence structures seemed to offer unlimited levels of complexity. Some authors had attempted to enumerate the number of requirement structures found but there was no reason to expect that there could be an upper limit, given the often-convoluted language constructs arising from committee and consensus authoring. It was necessary to separate the logical structure of normative documents from the interpretation of terms and exploit existing robust rule engines

The paradigm shift (Nisbet, 2008) came in recognising that the recursive structure of language and the roles of individual adjectives and nouns fell into four distinct categories. Operable knowledge occurs as words and sections applying, narrowing, broadening, and excluding scope. The application to the scope can be normative, definitive or descriptive.

The four categories transcend language, grammar and style. The skill to recognise the categories in the source text can be found in the regulatory experts who are most familiar with the text. The identified terms can be catalogued in a dictionary (strictly a thesaurus), along with explanatory plain language expressions to support any extended dialogue

1 Director, AEC3 Uk Ltd, London, UK, nn@aec3.com,

PhD student, Leeds Beckett University, UK, n.nisbet8827@student.leedsbeckett.ac.uk 
with applicants. Technical objects and query strings applicable to the descriptive resources such as reports or BIM models can be added.

\section{RESEARCH FINDINGS}

The novelty of this conceptualisation of knowledge represents a challenge to the current assumptions in this sector and beyond. The challenge has been met in two ways: firstly, by indicating a theoretical basis, and secondly by showing its application in practice.

The four-fold analysis can be analogised with the Aristotelian square-of-opposition terms used for predicate logic (Hjelseth, 2011). It can be associated to the four operators found in computer logic ('AND', 'OR', 'NOTAND' and 'NOTOR'). Taken together these create an assurance the conceptualisation is complete and effective. Other logical operators that are included in the literature of logic such as 'exclusive-or' and 'if-and-only-if' can be represented using the four categories when written out in full plain text and marked-up.

The methodology has been exploited in a number of demonstrations and trials. A clause of the US ICC IECC was captured and delivered as a resource for three distinct checking engines within a few weeks of the conceptualisation being announced. Large tables of requirements for the US NIBS WBDG hospital design guide were captured and checked against a reference hospital design. Suggested room-swops were checked as a design support tool. In 2016 the UK 'InnovateUK RegBIM' project (Beach, 2013) showed that parts of UK Building regulations and the BREEAM environmental guidance scheme could be captured and checked against a BIM model. The mark-up was used to automatically generate intermediate representations, such as the IFC constraint schema and the Java Drools rule representation. Subsequently, SPARQL, DMN and Java/C++procedural-code representations have been generated automatically for use by other applications. In 2017 AEC3 began development of a workbench using the source marked-up text directly. This is now operational and offers the opportunity for inclusion of features not always available through other rule-engines such as heuristics, tracking of unknowns, and the introduction of uncertainty.

A third response showing that this work can 'move-the-dial' of the perception of automated code compliance amongst UK construction experts began last year with the authoring and collation of an industry-wide questionnaire under the UK CDBB DCOM initiative. These results will form the baseline for specific trials next year.

\section{REFERENCES}

Nisbet N, Wix J and Conover D. 2008. "The future of virtual construction and regulation checking", in Brandon, P., Kocaturk, T. (Eds), Virtual Futures for Design, Construction and Procurement, Blackwell, Oxfordshire. doi: $10.1002 / 9781444302349 . \operatorname{ch} 17$

Wix, J., Nisbet, N. and Liebich, T. 2008. "Using Constraints to Validate and Check Building Information Models" 7th European Conference on Product and Process Modeling (ECPPM) Sophia Antipolis. 10.-12 Sept. 2008 p. $467-$ 476 Edited by Raimar Scherer and Alain Zarli Taylor \& Francis 2008. Print ISBN: 978-0-415-48245-5. eBook ISBN: 978-0-203-88332-7

Eilif Hjelseth, Nick Nisbet (2011) "Capturing normative constraints by use of the semantic mark-up (RASE) methodology” in CIB W78 2011 28th International Conference - Applications of IT in the AEC Industry.

Beach TH, Kasim T, Li H, Nisbet N, Rezgui Y, "Towards automated compliance checking in the construction industry”, Lecture Notes in Computer Science, 8055 (2013) 366-380 ISSN 0302-9743 10.1007/978-3-642-40285$2 \_32$ 